\title{
LA TRANSFORMACIÓN CONCEPTUAL DEL MODELO POLÍTICO CLÁSICO DEL ESTADO EN TOMÁS DE AqUINO*
}

\author{
The Conceptual Transformation \\ of the Classic Political Model of \\ the State in Thomas Aquinas*
}

\author{
A TRANSFORMAÇÃO CONCEITUAL \\ DO MODELO POLÍTICO CLÁSSICO DO \\ Estado EM TomÁs de Aquino*
}

* Las ideas expuestas en este trabajo son fruto de las enseñanzas, el diálogo y la discusión generadas en el seminario "Ruptura medieval del modelo político clásico e irrupción de la teoría política”, dictado por el Dr. Francisco Bertelloni en la Facultad de Filosofia y Letras de la Universidad de Buenos Aires (UBA), durante el 2016. / The ideas in this work are the result from to the teachings, dialogues and discussions around the workshop "Ruptura medieval del modelo político clásico e irrupción de la teoría política" given by the Dr. Francisco Bertelloni in the Phylosophy Faculty of the Universidad de Buenos Aires, through 2016. / As ideias expostas neste trabalho são fruto dos ensinamentos, do diálogo e da discussão gerados no seminário "Ruptura medieval del modelo político clásico e irrupción de la teoría política”, ministrado pelo doutor Francisco Bertelloni, na Faculdade de Filosofia e Letras da Universidad de Buenos Aires (UBA), durante 2016.

** orcid.org/0000-0003-3182-5219. Universidad de Buenos Aires (UBA), Argentina. eduardomagoja@derecho.uba.ar

RECIBIDO: 22/1 1/2018. ENVÍO A PARES: 22/11/2018

APROBADO POR PARES: 25/11/2018. ACEPTADO: O7/12/2018

DOI: $10.5294 /$ DIKA.2019.28.2.5

PARA CITAR ESTE ARTÍCULO / TO REFERENCE THIS ARTICLE / PARA CITAR ESTE ARTIGO EDUARDO ESTEBAN MAGOJA, "LA TRANSFORMACIÓN CONCEPTUAL DEL MODELO POLÍTICO CLÁSICO DEL ESTADO EN TOMÁS DE AQUINO”, EN DÍKAION 28, 2 (2019), 333-354. 


\section{RESUMEN}

En términos conceptuales, la historia de la teoría politica presenta, grosso modo, dos grandes modelos explicativos acerca del surgimiento y los fines del Estado. Por un lado, un modelo clásico representado por Platón, Aristóteles y Cicerón, que se caracteriza por el propósito de lograr la conformación de hombres virtuosos. Por otro lado, un modelo propio de la Modernidad, que concibe el Estado como una consecuencia para satisfacer las necesidades de la vida, solucionar los conflictos entre los particulares y garantizar su preservación. Entre ambos momentos históricos (Antigüedad y Modernidad), el pensamiento político medieval esconde la llave para poder comprender ese viraje. De hecho, tal aspecto fue advertido hace tiempo por Arendt, Habermas y, mucho más recientemente, por Bertelloni. Focalizados en esta temática, este trabajo tiene el propósito de analizar cómo se presenta en Tomás de Aquino ese desplazamiento a partir de una (in) consciente resemantización del concepto de naturaleza (phýsis/natura), y también a raíz de la emergencia de un contexto de enunciación especial que posibilitó la novedad.

\section{PALABRAs CLAVE}

Estado; Tomás de Aquino; modelo político clásico; modelo político moderno; naturaleza. 


\section{ABSTRACT}

From a conceptual point of view, the history of political theory presents grosso modo two great models which explain the emergence and the aims of the State. On the one hand, a classic model, represented by Plato, Aristotle and Cicero, which is characterized by the purpose of achieving the conformation of virtuous men. On the other hand, a modern model, which understands the State as a consequence to satisfy the necessities of life, to solve conflicts between individuals and guarantee their preservation. Between both historical moments (antiquity and modernity), the medieval political thought hides the key to understand that change. In fact, this was perceived some time ago by Arendt, Habermas and much more recently by Bertelloni. Focused on this topic, this paper has the purpose of analyzing how the shift appears in Thomas Aquinas as a result of an (un)conscious resemantization of the concept of nature (phýsis/natura) and also the emergence of a special context of enunciation that made possible the novelty.

\section{KEYWORDS}

State; Thomas Aquinas; classical political model; modern political model; nature. 


\section{RESUMO}

Em termos conceituais, a história da teoria política apresenta, de modo geral, dois grandes modelos explicativos sobre o surgimento e os objetivos do Estado. Por um lado, um modelo clássico representado por Platão, Aristóteles e Cícero, que é caracterizado pelo propósito de conformar homens virtuosos. Por outro, um modelo próprio da Modernidade, que concebe o Estado como uma consequência para satisfazer as necessidades da vida, solucionar os conflitos entre os particulares e garantir sua preservação. Entre ambos os momentos históricos (Antiguidade e Modernidade), o pensamento político medieval esconde a chave para poder compreender essa virada. De fato, esse aspecto foi advertido faz tempo por Arendt, Habermas e, muito mais recentemente, por Bertelloni. Focados nessa temática, este trabalho tem o objetivo de analisar como é apresentado, em Tomás de Aquino, esse deslocamento a partir de uma (in)consciente ressemantização do conceito de natureza (phýsis/natura), e devido à emergência de um contexto de enunciação especial que possibilitou a novidade.

\section{PALAVRAS-CHAVE}

Estado; Tomás de Aquino; modelo político clássico; modelo político moderno; natureza. 
SUMARIO: INTRODUCCIÓN. 1. EL MOdELO POLÍtICO CLÁSICO DEL ESTADO. 1.1. LA VIDA VIRTUOSA EN LA REPÚBLICA DE PLATÓN. 1.2. LA KOINONÍA TÉLEIOS Y LA ARETÉ CIUDAdANA EN ARISTÓTELES. 1.3. EL NATURALISMO POLÍTICO EN DE FINIBUS BONORUM ET MALORUM DE CICERÓN. 2. LA CONFIGURACIÓN DEL REGNUM EN EL PENSAMIENTO POLÍTICO DE TOMÁS DE AQUINO. 3. LA FILOSOFÍA POLÍTICA DE TOMÁS DE AQUINO SEGÚN LA LECTURA DE ARENDT Y HABERMAS. 4. DE LA PHÝSIS ARISTOTÉLICA A LA NATURA TOMISTA.

5. CONCLUSIONES. BiBLIOgRAFía.

\section{INTRODUCCIÓN}

Si observamos la historia de la teoría política desde la filosofia griega del siglo IV a. C. hasta la Modernidad, se puede advertir la existencia de dos grandes modelos explicativos distintos acerca del surgimiento y los fines del Estado. En un extremo, autores tales como Platón, Aristóteles y Cicerón son grandes representantes de un modelo clásico, el cual se caracteriza por poner un fuerte acento en el desiderátum de lograr un hombre virtuoso. En el otro extremo, en la Modernidad encontramos un modelo político que acentúa las necesidades de la vida y la conservación individual, y que ve el Estado como un efecto para neutralizar los conflictos entre los particulares. Así, por ejemplo, en Locke la sociedad civil se establece con el fin de garantizar el derecho de los individuos como propietarios, ${ }^{1}$ y en el caso de Hobbes el Leviatán se erige en vistas de la conservación individual. ${ }^{2}$

El pensamiento político medieval del siglo XIII es un campo privilegiado para comprender cómo se produjo ese desplazamiento. En efecto, en los textos de esta época se puede ver que conviven tanto el modelo del Estado virtuoso (aristotélico-estoico) como también un modelo protomoderno, cuya tensión revela la existencia de una resemantización de las categorías políticas clásicas que posibilitó la apertura a la teoría política moderna. Así, dentro de los estudios políticos, Arendt y Habermas han identificado a Tomás de Aquino como el principal responsable de la reconfiguración conceptual. ${ }^{3}$ A grandes rasgos sostienen que el filósofo medieval, en su obra De regno, desplaza el esquema ético-metafísico aristotélico, traslada las relaciones propias de la familia (oîkos) al orden estatal, y define el regnum como el espacio para satisfacer las necesidades inmediatas. Focalizado en este tema, Bertelloni ha dado cuenta, en una reciente investigación, que la cuestión es mucho más compleja y que en ese proceso intervinieron aun con mayor intensidad autores tales como Juan de París, Marsilio de Padua y Nicolás de Cusa. ${ }^{4}$

1 Cf. John Locke, Second Treatise of Government, Indianapolis, C.B. Macpherson, 1980 (primera edición 1690), cap. IX.

2 Cf. Thomas HobBes, Leviatán: o la materia, forma y poder de una república eclesiástica y civil, Buenos Aires, FCE, 2007 (primera edición 1651). En tal sentido se expresa en especial en el capítulo XVII.

3 Hannah Arendt, La condición humana, Buenos Aires, Paidós, 2009 (primera edición 1958), p. 38; Jürgen Habermas, Teoria y praxis, Madrid, Tecnos, 1987, pp. 54-57.

4 Cf. Francisco Bertelloni, "Facere de necessitate virtutem. El principio conservatio sui en la teoría politica medieval”, en Devs mortales 11 (2015), pp. 9-39. 
Ahora bien, en este trabajo se retomarán las ideas trazadas por estos autores en relación con Tomás de Aquino a los efectos de indagar cómo en la pérdida del sentido originario de las categorías politicas clásicas hay una resignificación del concepto de naturaleza griega (phýsis), el cual deja de hacer referencia al hombre virtuoso y expresa, en cambio, una natura falente. También veremos, aunque con brevedad, cuáles fueron las condiciones de posibilidad histórico-políticas que permitieron aquel cambio. En el desarrollo de este tópico seguiremos de cerca los pasos trazados por Bertelloni, ${ }^{5}$ cuyos aportes al tema son una guía imprescindible para no extraviarnos en la compleja interpretación de los textos políticos medievales.

Se seguirá el siguiente orden de exposición con el fin de ganar claridad y rigor argumentativos. En primer lugar, se expondrán los lineamientos básicos del modelo político clásico, mediante una breve explicación de Platón, Aristóteles y Cicerón. ${ }^{6}$ En segundo lugar, se estudiará cómo Tomás de Aquino aborda el problema del poder temporal, y cómo ello ha sido interpretado por Arendt y Habermas en relación con el tránsito hacia un modelo político moderno. En tercer lugar, siguiendo a Bertelloni, veremos la resemantización del concepto de naturaleza aristotélico que se puede advertir en el filósofo medieval y el contexto de discusión en el que ello se formula.

\section{El MOdELO POLÍTICO CLÁSICO DEL ESTAdo}

\subsection{La vida virtuosa en la República de Platón}

El primer gran exponente de la teoría política clásica es Platón, de quien nos interesa destacar algunas ideas que expone en República. Vale aclarar que, si bien este diálogo no fue conocido en Occidente sino recién en el siglo $\mathrm{XV},{ }^{7}$ su espíritu (la idea de hacer al hombre virtuoso) se corresponde con el pensamiento de su discípulo Aristóteles y, por eso, es importante tenerlo en cuenta. ${ }^{8}$

En el libro II, Platón traza un boceto de una ciudad primitiva en la que prima la satisfacción de necesidades. ${ }^{9}$ En este modelo, ningún individuo se basta a sí mismo y las distintas necesidades lo llevan a asociarse con otros, a reunir "en una sola

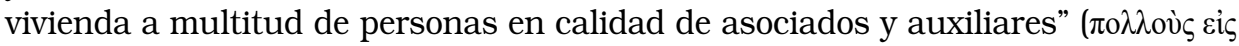

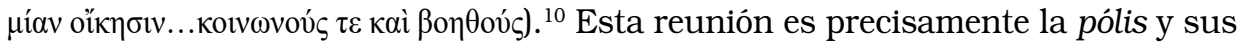

$5 \quad$ En especial dos trabajos: Francisco Bertelloni, "Natura multipliciter dicitur. Variantes en el uso de natura en la teoría política medieval a partir de la segunda mitad del siglo XIII", en Scripta 4 (2) (2011), pp. 11-29, y Francisco Bertelloni, "Facere de necessitate virtutem", ob. cit., pp. 9-39.

6 En lo que respecta a las fuentes griegas y latinas se han utilizado las ediciones o traducciones consignadas en la bibliografia en el apartado correspondiente.

7 Cf. Ernest Fortin, "St. Thomas Aquinas", en Leo Strauss y Joseph Cropsey (eds.), History of Political Philosophy, Chicago, University Press, 1987, p. 249.

8 No obstante, no se pueden desconocer las serias críticas formuladas por Aristóteles. Sobre el tema 338 nos remitimos al trabajo de Robert Mayhew, Aristotle's Criticism of Plato's Republic, Lanham, Row9369 b y ss.

$10369 c$. 
cimientos son las necesidades humanas, que van desde la provisión de alimentos, vivienda y vestido, hasta otros bienes y artículos que son provistos por quienes se dediquen a un oficio determinado. ${ }^{11}$

Sin embargo, en Platón las necesidades no agotan el sentido de la política, aunque es un elemento importante. En efecto, los ciudadanos organizados con base en el principio de división de funciones o de trabajo producirán trigo, vino, vestidos y zapatos; construirán viviendas, trabajarán y se alimentarán. ${ }^{12}$ Tendrán sus momentos de ocio, beberán vino y, entre otras cosas, disfrutarán de grandes manjares. Pero luego de vivir en paz morirán y les dejarán a sus descendientes un tipo de vida similar a la de ellos. Todo esto no es más, como le indica Glaucón a Sócrates, que "una ciudad de cerdos" (vंõv $\pi$ ó $\lambda v v) .{ }^{13}$

Platón sostiene que en realidad la ciudad ideal ("la pintura más hermosa de Gobierno") debe promover la virtud (areté) y para ello es fundamental que la filosofía y la política vayan de la mano. ${ }^{14}$ Así, en la Carta VII explica que los males del género humano no cesarán hasta que el poder sea ocupado por "la raza de los fi-

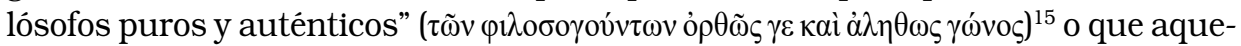
llos que gobiernen la ciudad lleguen a ser filósofos verdaderos. Esta misma tesis es presentada en República con un mayor desarrollo. ${ }^{16}$ Platón despliega un largo razonamiento a los efectos de determinar quiénes son esos verdaderos filósofos, ${ }^{17}$ y sostiene que no son aquellos que se extravian en la multitud de las cosas, sino los que pueden alcanzar "lo que siempre se mantiene igual a sí mismo" (

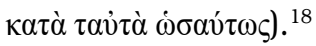

Los auténticos filósofos poseen el verdadero conocimiento, es decir, la epistéme, pues conocen las Ideas y entre ellas la Idea suprema de Bien. ${ }^{19}$ Esto los habilita a ejercer el gobierno, pues su conocimiento y su virtud es lo que va a garantizar la buena organización de la ciudad y que los hombres vivan bien. En este sentido, no se debe perder de vista que el planteo político platónico gira en torno a la pregunta acerca de la justicia (dykaios ne), con el propósito de ofrecer una respuesta sobre el mayor grado de felicidad que los ciudadanos pueden alcanzar en el seno de la pólis. Así pues, como explica Soares, Platón, en su proyecto, no deja de insistir

$11369 \mathrm{c}$

$12372 \mathrm{a}-372 \mathrm{c}$.

13372 d.

14 Existen ciertas discrepancias en la literatura acerca de cuál es el modelo de pólis ideal en Platón, pues a lo largo de su obra presenta tres: una sana, una injusta y una purificada. Más allá de los problemas interpretativos está claro que no es en la "ciudad de cerdos" donde se encuentra el modelo político realmente deseable, sino que este exige un componente virtuoso que vaya más allá de las simples necesidades humanas. Sobre el tema, ver Claudia Mársico, "El status de la pólis sana en La República de Platón", en Diálogos 74 (1999), pp. 149-167.

$15326 \mathrm{~b}$.

$16 \quad 473 \mathrm{e}-474 \mathrm{a}$.

17 474b-480a.

$18484 \mathrm{~b}$.

19 Toda la filosofia política de Platón se articula en torno a la teoría de las Ideas, la cual brinda una fundamentación metafísica que aparece cristalizada en la figura del filósofo rey (Lucas SoAREs, Platón y la política, Madrid, Tecnos, 2010, pp. 161-174). 
en la eudaimonía entendida como "vivir bien" ( $\varepsilon \tilde{j} \zeta \tilde{\eta} v)$, que es la meta que debe lograr la politica. ${ }^{20}$ Este componente ético se resume muy bien en Leyes, cuando el Ateniense se pregunta cómo podría habitarse del mejor modo una ciudad y "cómo

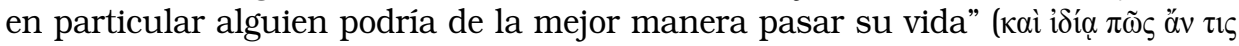

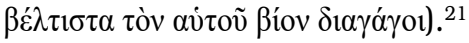

\subsection{La koinonia téleios y la areté ciudadana en Aristóteles}

El panorama platónico acera de la buena vida se repite en Aristóteles. Basta con analizar el libro I de la Ética Nicomáquea y el libro I de la Politica para ver cómo es presentada la idea. En el comienzo de la primera obra mencionada aparece la teleologia aristotélica cuando dice que todo arte y toda investigación, toda acti-

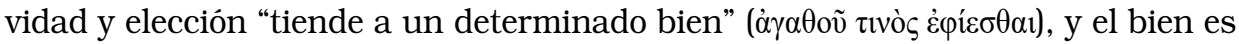

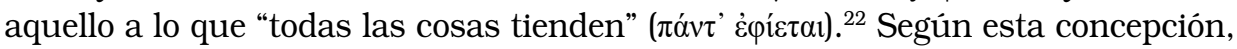
cada disciplina tiene un fin (télos) propio y los fines de las disciplinas gobernadoras tienen fines mayores. Hay fines intermedios y fines últimos, y aquel que exis-

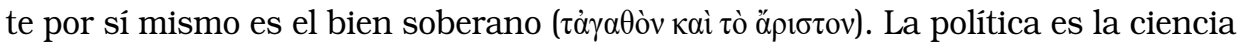
arquitectónica que se ocupa del fin último que ordena todos los fines anteriores, el cual es precisamente la eudaimonía. ${ }^{23}$

Queda claro que en esta primera parte de la Ética se puede ver que la política es racionalidad y eticidad. En cuanto eticidad es una ciencia práctica a la cual están subordinadas la estrategia, la retórica y la economía, y en cuanto racionalidad engloba a las demás ciencias prácticas y favorece la realización racional del hombre: solo recién en la pólis este se desarrolla como tal y alcanza su télos.

En el libro I de la Política Aristóteles vuelve a presentar a la política como ciencia arquitectónica y, además, se ocupa de desarrollar el estatus ontológico de la pólis y la diferencia de cada comunidad (koinonía). ${ }^{24}$ En su exposición, Aristóteles aplica el método analítico y sigue dos criterios para cualificar cada koinonía: el télos y el tipo de dominio. En relación con el primero, dice que toda comunidad tiende a un bien y ese bien es el fin, que es determinante en la ontología de la comunidad. Tanto la casa (oîkos) como la aldea (kóme) tienen un fin, pero la ciudad (pó-

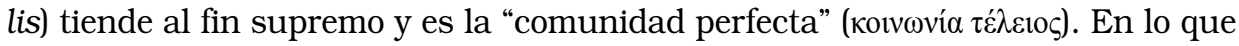
respecta al dominio, va a mostrar que en cada comunidad existe una diferencia acerca de su gobierno: mientras que en el oîkos y la aldea hay verticalidad, la pólis se caracteriza por su horizontalidad.

En la exposición acerca de la pólis, Aristóteles entremezcla una visión de corte histórico-genético y otra de tipo ontológico. En términos cronológicos dice que primero se constituye la casa, en donde el patér ejerce el dominio vertical al

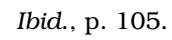


igual que un rey. Esta comunidad tiene como fin la satisfacción de necesidades primeras, cotidianas e inmediatas. En segundo término, a partir de una multitud de casas se constituye la aldea, la cual satisface necesidades no cotidianas. Existe también aquí un dominio vertical: por eso Aristóteles dice que estuvieron al principio gobernadas por reyes. La pólis es presentada al final del proceso y constituye la koinonía téleios. Tiene el limite de la total autosuficiencia y existe con el fin del "vivir bien" ( $\varepsilon \tilde{\tilde{\zeta}} \zeta \tilde{\eta} v)$, no la simple vida. Esto supone un componente virtuoso que puede atarse a la felicidad de la que Aristóteles habla en la Ética Nicomáquea. El buen vivir es aquello hacia lo cual tienden la casa y la aldea y, por

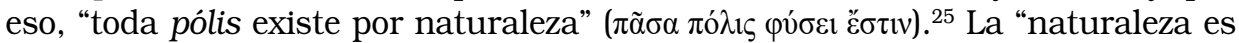

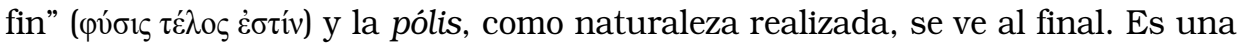
naturaleza que implica a las comunidades anteriores, de modo que no es posible disociar el proceso de las necesidades, del proceso que explica su aparición. Se puede decir, pues, que las comunidades son politicas en el sentido de que no pueden ser separadas del orden de la politicidad.

Tras desarrollar la idea del hombre como "animal político por naturaleza" ( $\varphi$ бøє

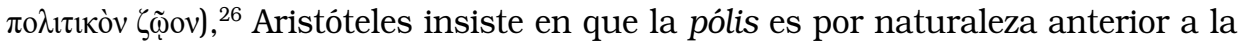
casa y a cada uno de los individuos. ${ }^{27}$ En esta instancia privilegia una perspectiva ontológica, pues dice que en el orden del ser la pólis es primera, es decir, anterior al proceso de desarrollo de las comunidades prepoliticas y constituye la causa final de estas comunidades. Así, el todo es anterior a las partes y la pólis es un todo orgánico que lo abarca todo. Este sistema metafísico aristotélico, como se puede advertir, hace dificil considerar separadamente la pólis de las partes y, más aún, las partes separadas de ella, de manera que la ontologia politica prima por sobre la cronologia de la ciudad.

Hay un último pasaje de la Politica que es importante señalar. En el libro III, Aristóteles presenta la pólis como la comunidad de libres e iguales y como el lugar de realización de la vida ciudadana. Allí vuelve a decir que los hombres no se han asociado para vivir, sino para vivir bien. ${ }^{28}$ Descarta la riqueza, la simple vida, el comercio, el socorro y la guerra como motivos de la conformación de la ciudad. La pólis que verdaderamente es (no de mero nombre) es aquella que procura la virtud; de no ser así, sería una alianza que solo se diferenciaría de otras por estar en un mismo lugar y en la que la ley es un convenio incapaz de volver a los ciudadanos justos. ${ }^{29}$ Pues bien, en estas alianzas en las cuales los hombres se acercan unos a otros por comercio, riqueza o guerra, los aliados son lejanos. Y alli la ley se transforma en un contrato, es decir, en una garantía de los derechos individuales vacía de moralidad.

25 Al respecto, ver Christopher ReEve, "The naturalness of the polis in Aristotle", en Georgios AnaGnostopoulos (ed.), A Companion to Aristotle, Oxford, Wiley-Blackwell, 2009, pp. 512-525; Timothy Chappell, "Naturalism' in Aristotle's political philosophy”, en Ryan Balot (ed.), A Companion to Greek and Roman Political Thought, Oxford, Wiley-Blackwell, 2009, pp. 382-398.

26 Ver al respecto Wolfgang Kullmann, "Man as a political animal in Aristotle”, en David KeYT y Fred Miller, Jr. (eds.), A Companion to Aristotle’s Politics, Oxford, Blackwell, 1991, pp. 94-117.

$27 \quad 1253 a 20$ y ss.

28 1280a.

$291280 \mathrm{~b}$. 


\subsection{El naturalismo politico en De finibus bonorum et malorum de Cicerón}

El último exponente del modelo político clásico que nos queda por presentar es Cicerón, cuyo naturalismo también influyó en Tomás de Aquino. El proyecto estoico ciceroniano se puede dividir en dos partes que guardan una relación de continuidad. En primer lugar, hay un momento en que el hombre está movido por la fuerza de la necesidad y de la autopreservación. Se trata de la doctrina estoica de la oikeiosis, ${ }^{30}$ que los latinos entendieron como una inclinación natural y teleológica del hombre hacia su propia conservación. ${ }^{31}$ En este sentido, Cicerón dice que "el primer deber" (primum est officium) consiste en que el hombre se conserve en su "constitución natural" (naturae statu). ${ }^{32}$

De acuerdo con la oikeíosis, los hombres y los animales tienen una disposición natural hacia su propio cuidado y a procurarse todo aquello que sea necesario para su subsistencia. Es un momento en el que predomina el bios, es decir, la vida. Así, Cicerón sostiene que desde el instante en que el animal nace se siente atraído hacia sí mismo y busca su propia conservación, ya sea mediante la obtención de bienes o el rechazo de todo aquello que pueda llevarlo a su destrucción. Este es el principio rector que rige la constitución natural de los seres vivos y es denominado "amor de sí mismo" (se diligendo). ${ }^{33}$

El niño, quien no ha desarrollado la plena racionalidad, se guía en función de esa inclinación a autoconservarse. Sin embargo, cuando irrumpe la racionalidad, hay una conversión del hombre y ese inicial amor de sí mismo se transforma en un appetitus societatis. En este segundo momento, el hombre declina su tendencia egoísta y la suplanta por una tendencia solidaria. En efecto, el appetitus societatis supone un viraje de la concepción del egoísmo hacia una concepción virtuosa y racional. El amor por los otros es una atribución al hombre de una evolución en la cual la racionalidad es la protagonista del cambio. A partir de esta transformación, el hombre toma consciencia de que es parte del mundo y antepone el beneficio común al interés individual y personal. ${ }^{34}$

Tal doctrina sigue de cerca a Aristóteles en su discurso histórico-genético y ontológico de la pólis, pues hay una serie de elementos en este segundo momento ciceroniano que habla de una racionalidad compartida que deja atrás la necesidad. De acuerdo con esta concepción, pues, el hombre tiene una disposición natural a

30 Sobre la oikeiosis en general, ver Troels ENGBERG-PEdersen, The Stoic Theory of Oikeiosis: Moral Development and Social Interaction in Early Stoic Philosophy, Aarhus, University Press, 1991. En lo que respecta a esta doctrina en el pensamiento ciceroniano ver los trabajos de Magnaldi GrusePPINA,

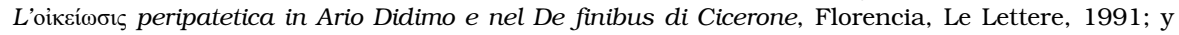
también Laura Corso de Estrada, "Oikeíosis. Ciceronian Reading and XIII Century Receptions", en Alejandro VIGo (ed.), Oikeiosis and the Natural Basis of Morality. From Classical Stoicism to Modern Philosophy, Hildesheim, G. Olms, 2012, pp. 67-94. 
asociarse. Es el único de los seres vivos que "tiene el apetito de uniones y sociedad de hombres" (appetensque convictum hominum ac societatem). ${ }^{35}$ De hecho, utilizando un ejemplo de Aristóteles, Cicerón sostiene que esta unión, si bien se da entre las abejas y las hormigas, es mucho más fuerte en los hombres, pues de acuerdo con su disposición natural existen en vistas a formar comunidades y sociedades. ${ }^{36}$

\section{LA CONFIGURACIÓN DEL REGNUM EN EL PENSAMIENTO POLÍTICO DE TOMÁS DE AQUINO}

Hemos visto el modelo político clásico mediante una breve exposición del pensamiento de Platón, Aristóteles y Cicerón. Si nos detenemos en especial en estos dos últimos autores, se puede advertir que ambos, como explica Bertelloni, "invocan la teleología de la natura como principio del movimiento autoperfectivo de los seres" ${ }^{37}$ Sin embargo, existen diferencias entre ellos, sobre todo acerca de qué se entiende por naturaleza. No me interesa detenerme en este aspecto, pero sí destacar dos puntos que merecen atención. En primer lugar, que en el modelo estatal aristotélico y ciceroniano el orden político está ligado con la virtud. Ninguno de los dos transforma en lema la necesidad, sino que la virtud la envuelve. En segundo término, considero importante señalar que el texto aristotélico y el ciceroniano se pueden leer desdoblando el discurso en dos partes: una negativa que se caracteriza por la necesidad de pasar a otro estadio; otra positiva cuyas notas distintivas son la virtud y la racionalidad. Este segundo punto es el más importante, pues al parecer los autores medievales leyeron los textos clásicos de esta manera y acentuaron el estadio de falencias. Con la ayuda de Arendt y Habermas veremos que eso es lo que sucede en Tomás de Aquino. Pero la exposición exige que presentemos primero dos textos en donde el filósofo medieval expone su pensamiento politico: la Summa Theologiae y, principalmente, el De regno.

De la primera de estas obras es importante analizar el artículo 4 de la quaestio 96 de la prima pars. ${ }^{38} \mathrm{Si}$ bien el texto es breve, resulta muy esclarecedor y anticipa algunas ideas que desarrollará con mayor profundidad en el De regno. El problema que guía la reflexión tomista es si "en el estado de inocencia había dominio del hombre al hombre” (in statu innocentiae homini dominabatur). Tres son las objeciones que Tomás de Aquino advierte que se pueden hacer para rechazar esa idea: en primer lugar, San Agustín dijo que Dios creó al hombre como un ser racional, que solo es señor de los seres irracionales, situación que invalida la posibilidad de relaciones de dominación; en segundo lugar, se sostiene que el dominio es consecuencia del pecado y solo se da una vez producida esta ruptura de la naturaleza humana; en tercer término, se dice que el sometimiento y la libertad se oponen, y puesto que en el estado de inocencia todos eran libres no podia haber dominio.

\footnotetext{
35 De finibus, IV, 18

36 De finibus, III, 63.

37 Francisco Bertelloni, "Facere de necessitate virtutem", ob. cit., p. 17.

$38 \mathrm{Al}$ respecto, ver Francisco Bertellon, “¿Qué significa 'política' en STh. I, q. 96, a. 4? Sobre la génesis de la semántica de un vocablo”, en José Meirinhos y Olga WeiJers (eds.), Florilegium mediaevale. Études offertes à Jacqueline Hamesse à l'occasion de son éméritat, Louvain-La-Neuve, 2009, pp. 19-37.
} 
En contra de estos argumentos, Tomás de Aquino quiere explicar que la naturaleza humana en el estado de inocencia ya era política. Si el hombre pasó del estado de inocencia al estado pospecado, lo hizo cargado con sus características originarias y dentro de ellas se encuentra el ejercicio de dominio. La solución que propone se monta, en primer término, sobre una distinción. Hay un sentido de dominio que no se da en el estado de inocencia, que es el que está ligado a la servidumbre. Pero hay otro sentido que sí era posible: el de la "dirección de personas libres" (dirigendi liberos). Así, el dominio no contradeciría la naturaleza humana en el estado de inocencia y podría haberse dado.

La posibilidad de su existencia se funda en que el dominio entre libres coopera al bien del dirigido y al bien común. Tomás de Aquino ofrece dos motivos que justifican por qué podia haber existido. El primero es que en el estado de inocencia "el hombre es por naturaleza un animal social" (quia homo naturaliter est animal sociale), y en ese estado vivia en sociedad. A la naturaleza humana le corresponde, entonces, una nota que es la sociabilidad. Ahora bien, ello no se da si no hay alguien que esté al frente y guie a los hombres, pues la multitud tiende a muchas cosas y requiere de uno que ejerza la dirección para que no se disperse. Es aquí donde introduce el segundo motivo, que tiene que ver con la mayor capacidad de algunos en ciencia y sabiduría, cuyos beneficios deben estar puestos al servicio de los demás.

Al exponer estos argumentos, Tomás de Aquino introduce una falencia que trata de remediar con el dominio. En efecto, la sociabilidad implica la posibilidad de la dispersión, de manera que la unidad de la multitud solo puede ser construida con el dominio. Lo interesante de todo esto es que explica la relación de dominio en virtud de una situación negativa anterior, y abre la posibilidad de leer la política como consecuencia de un estado de necesidad. En la visión tomista la naturaleza humana tiene dos características, que son la sociabilidad y la politicidad, y la segunda tiende a remediar los defectos que puede tener la primera.

En el De regno, escrito entre 1271 y 1273, Tomás de Aquino ofrece un desarrollo mucho más profundo de la cuestión de la sociabilidad y la politicidad que fue presentada en la Summa Theologiae. Veamos cómo presenta sus ideas.

En el prólogo se aclaran cuáles van a ser los dos grandes temas que se propone abordar: por un lado, el origen del Estado basado en la necesidad; por el otro, aquello que atañe al deber del rey en tanto epígono del poder espiritual. En el desarrollo de estos temas hay tres aspectos del libro I que resultan relevantes. El primero es la conformación de la vida de los hombres con otros, es decir, la sociedad. En este sentido, sigue la concepción finalista de Aristóteles según la cual la naturaleza tiene un fin y el fin supremo del hombre en la tierra es la felicidad. ${ }^{39}$

39 Summa Theologiae, I-II, q.2 a.7, q.3 a.1, q.69 a.1. 
El hombre tiene en su interior la luz de la razón, mediante la cual dirige sus actos hacia ese fin. Sin embargo, en su realización se puede extraviar y, por eso, en la vida con otros precisa de un guía. En este contexto, dice que el hombre es un "animal politico y social" (animal sociale et politicum), que "vive en multitud" (in multitudine vivens). El impulso que lo lleva a vivir en comunidad es la necesidad natural. Esto lo justifica al explicar las carencias y falencias del hombre en comparación con otros animales, los cuales tienen pieles, colmillos, garras y velocidad para protegerse. Sin embargo, Tomás de Aquino va a sostener que el hombre es el único dotado de razón y mediante ella, y el officium manuum, puede procurarse de los recursos para su subsistencia. El uso efectivo de esta capacidad no lo puede realizar un solo hombre, sino que se necesita una vida in multitudine. De este modo, el hombre es movido por un impulso natural a constituir una sociedad. La argumentación tomista muestra, pues, que la vida en sociedad es consecuencia de necesidades y falencias, y su constitución obedece a una tendencia natural.

El segundo aspecto relevante es la forma en que Tomás de Aquino introduce el problema del dominio del rey sobre otros hombres. De acuerdo con los argumentos que ha trazado respecto a la constitución de la sociedad, justifica el gobierno de uno en virtud de las falencias que tienen los hombres en la vida en sociedad. En efecto, los hombres no privilegian el bien de todos, sino el bien personal. Esto llevaria a la sociedad a su destrucción, cuando en realidad a lo que se debe aspirar es hacia la conservación de la unidad social. Esta unidad se sostiene y preserva en la medida que haya un Gobierno que dirija a los hombres al bien común. Solo así el "régimen será recto y justo" (regimen rectum et iustum).

Hasta aquí se advierte que el regnum es un estadio que viene a suplir una situación de falencias. Es una continuación posterior a la sociedad, que se monta sobre una situación viciosa a los efectos de neutralizar sus aspectos negativos. Así, el rey tiene la función de conducir a los hombres hacia el bien común tras direccionar los intereses individuales. Incluso, como advierte Bertelloni, además de vincular el regnum con las deficiencias de la vida societaria, Tomás de Aquino identifica la "comunidad perfecta" (perfecta communitas) con la simple vida y la satisfacción de necesidades básicas. La ciudad es el espacio, pues, que permite lograr "lo necesario para la vida" (ad necessaria vitae).

En último término, no se puede dejar de señalar que esta posición se enmarca dentro de una concepción en la que Tomás de Aquino dice que el rey tiene la función de conducir al hombre hacia la virtud. En el libro II sostiene que "el último fin de una multitud congregada es vivir según la virtud” (videtur autem finis esse multitudinis congregatae vivere secundum virtutem). Pareciera ser que, si bien en la constitución de la vida social prima la necesidad, "la vida virtuosa" la dota de contenido moral en tanto se expresa como su fin soberano (la felicidad). ${ }^{40}$ Sin embargo, más

40 En tal sentido, Tomás de Aquino entiende la realización de la felicidad de modo equivalente a Aristóteles, es decir, como fin último. Sin embargo, la felicidad última no se realiza en el mundo terrenal, sino de modo sobrenatural, pero sigue siendo la consumación de una virtus (Francisco 
allá de que aquí Tomás de Aquino introduce la realización de la virtud de los hombres como fin de la sociedad, no logra conciliar este elemento con las falencias que motivan el origen del regnum. En realidad, la virtud ocupa un lugar secundario en la argumentación del tratado pues aquello que en verdad justifica el origen de la sociedad y la necesidad del rey es la naturaleza deficiente del hombre, no una "naturaleza teológica entendida como perfección" ${ }^{41}$

\section{LA FILOSOFÍA POLÍTICA DE TOMÁS DE AQUINO SEGÚN LA LECTURA DE ARENDT Y HABERMAS}

En el pensamiento político contemporáneo, Arendt y Habermas se han ocupado de estudiar cómo Tomás de Aquino, sobre todo en el De regno, invirtió el concepto aristotélico de comunidad política y le atribuyó un sentido de societas, vacío del elemento virtuoso. Veamos cómo lee cada uno de ellos ese tratado político.

Arendt se ocupó del tema en su magistral obra The Human Condition. Su estudio está estructurado por dos ideas conductoras: primero, la denuncia de la irrupción de la noción de societas como concepto que hace perder a la política tradicional el carácter virtuoso; en segundo lugar, la confusión entre pólis y oîkos, y la importancia de seguir distinguiendo ambas esferas.

En lo que respecta a la primera idea, Arendt dice que Tomás de Aquino tradujo el zôon politikón aristotélico como animal social, y cuando hizo esto utilizó la palabra socialis en términos muy técnicos y distintos a la concepción política del filósofo griego, pues "social" quiere decir que el hombre integra una vida en multitud gobernada por uno que lo hace de arriba para abajo. En esta "inconsciente sustitución de lo político por lo social" 42 predomina la idea de que los hombres viven con otros en virtud de sus limitaciones. Así, Arendt dice que "la natural y meramente social compañía de la especie humana se consideraba una limitación que se nos impone por las necesidades de la vida biológica". ${ }^{43}$ Tal tesis es precisamente la que sostiene Tomás de Aquino en el De regno: los hombres viven en conjunto debido a sus falencias, y esto es lo que se va a denominar politico por naturaleza.

En el pensamiento griego, la capacidad del hombre en la esfera pública es por completo distinta a la asociación natural que se da en el seno de la familia. Todo ciudadano pertenece a dos órdenes que se hallan en oposición: lo que es idión (lo propio) y lo koinón (lo común). En este sentido, el zôon politikón se oponía a la asociación de la familia y esta distinción es lo que no pudo mantener el concepto latino de socialis.

Bertelloni, "Algunas reinterpretaciones de la causalidad final aristotélica en la teoría política medieval”, Cadernos de Historia e Filosofia da Ciencia 15 (2) (2005), p. 351).

41 Francisco Bertelloni, "Facere de necessitate virtutem", ob. cit., p. 25.

42 Hannah ARENDT, La condición humana, ob. cit., p. 38.

43 Idem. 
En relación con la confusión entre lo político y lo doméstico, Arendt explica que los griegos dividian ambos planos y le asignaban características propias. El rasgo distintivo del oîkos era que los hombres vivian juntos en razón de sus necesidades: la comunidad natural es, pues, hija de la necesidad. En la esfera de la pólis, en cambio, regia la libertad, que era la condición esencial para la eudaimonía. Al mismo tiempo, la pólis se diferenciaba de la familia en que estaba integrada por iguales, mientras que en el campo doméstico no había libertad ni igualdad. ${ }^{44}$ Esto es lo que vino a alterar Tomás de Aquino, en tanto concibe a la sociedad como una gran familia en la que el Gobierno es vertical, ${ }^{45}$ y esta asociación se instaura con el objeto de contrarrestar las limitaciones y carencias del hombre. Así, la filosofia politica se transforma en filosofia económica. ${ }^{46}$

En Teoría y praxis Habermas siguió los pasos trazados por Arendt, pero fue un poco más lejos con su interpretación. No solo dice que Tomás de Aquino al introducir la palabra socialis desplaza el modelo político virtuoso y confunde pólis con oîkos. Sostiene también que aquel transforma el vínculo político en un vínculo despótico y convierte al Estado en un orden formal y policial, cuyo fin es la paz.

En efecto, Habermas dice que en Aristóteles la verdadera pólis debe apoyarse en la virtud de sus ciudadanos. ${ }^{47} \mathrm{Si}$ la política no se alimentara de la areté, la comunidad sería una mera alianza o una confederación. Esta symmakhía no es otra cosa que la societas y se expresa como un modelo en el que prima la necesidad: es el resultado de un contrato de aquellos que se juntan entre sí movidos por el propio interés. Es por completo lo opuesto a la koinonía politiké.

Aristóteles, dice Habermas, "imagina la ficción contractual de derecho privado", pero para negar ese modelo. ${ }^{48}$ Se rechaza porque en esta ficción hay una primacía de lo privado sobre lo público o, mejor dicho, lo público es una consecuencia de lo privado. A esto precisamente se opone Aristóteles. Si los ciudadanos fundan la comunidad con fines comerciales o bélicos, esta unión no debe entenderse como una pólis. En la mera alianza cada uno se maneja en un espacio público que carece de una verdadera unión comunitaria y considera la ciudad como si fuera su casa: hace de la política una oikonomía. Hay, pues, una confusión del oîkos con la pólis, dos ámbitos bien distintos que reflejan la contraposición de lo privado y lo público, lo particular y lo común. La pólis no puede ser entendida como una casa, pues en esta se privilegia el interés de cada uno y la autoridad es vertical. Nada más lejos que la pólis, que es el espacio de la libertad, de la racionalidad y donde la fuerza se ha declinado al esquema de poder horizontal.

44 Ibid., p. 44.

45 En relación con la tesis de Arendt, algunos autores advierten ciertas debilidades hermenéuticas acerca de la extensión del modus del dominio doméstico a la realidad social, como también sobre la reducción de lo político a lo familiar. Al respecto, ver el detallado estudio de Santiago ARGÜELLo, "El lenguaje de la causalidad de nuestras acciones: hacia una resignificación del dominus en Tomás de Aquino", Pensamiento 71 (267) (2015), pp. 565-586.

46 Hannah ARENDT, La condición humana, ob. cit., p. 47.

47 Jürgen Habermas, Teoría y praxis, ob. cit., pp. 54 y ss.

48 Ibid., p. 55. 
Uno de los últimos puntos interesantes que señala Habermas en su trabajo es la ambivalencia que hay en Tomás de Aquino. En efecto, por un lado, se sitúa en la tradición aristotélica. Cuando habla del fin del reino (no del origen) señala que los hombres no se asocian solo para satisfacer la vida, sino para alcanzar la virtud. ${ }^{49}$ Pero, por otra parte, se aparta de Aristóteles en tanto convierte el regnum en una gran familia en la que el rey gobierna a sus súbditos como el padre a sus hijos, es decir, de un modo monárquico. ${ }^{50}$ En este sentido, en el campo político Tomás de Aquino edifica un orden vacio de justicia y de virtud, y puesto que se privilegia ese orden se pierde la participación de los ciudadanos en la administración y la legislación. Su postura privilegia la tranquilidad y la paz, fines que vienen a constituir el bien de la ciudad. Se trata, pues, de una mirada policial. ${ }^{51}$

\section{DE LA PHÝSIS ARISTOTÉLICA A LA NATURA TOMISTA}

Tanto Arendt como Habermas tienen el mérito de haber demostrado que en la concepción estatal de Tomás de Aquino se prescindió de la consideración de la pólis como un espacio virtuoso. El Estado es un artificio, una creación posterior que se presenta como una continuación de la naturaleza, pero que se construye para salvar las falencias del ser humano. Así pues, las relaciones oikonómicas entre los individuos es lo que genera lo público como una invención.

Sin negar el valor de esa lectura, ninguno de los dos autores parece advertir una cuestión todavía más fundamental: la resemantización del concepto de phýsis aristotélico. ${ }^{52}$ Este cambio fue posible en virtud de una reconfiguración del leguaje político y la emergencia de nuevos problemas teóricos, los cuales permitieron la apertura a otros horizontes de sentido.

En efecto, el naturalismo político de Aristóteles tiene una serie de presupuestos metafísicos y físicos, sin los cuales pierde su densidad teórica. Ya hemos visto

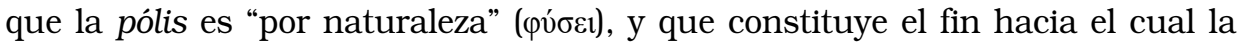
familia y la aldea tienden; sin embargo, esta idea que expone Aristóteles en la Politica se entiende mejor a la luz de algunas nociones generales de la Metafisica y la Física. De la primera de estas obras resulta interesante destacar la sexta definición de phýsis que analiza Aristóteles, según la cual dice que es "la entidad

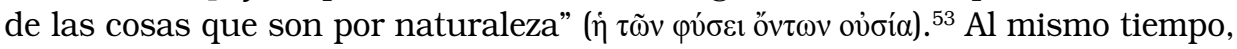
aclara que las cosas no poseen naturaleza hasta tanto alcancen "la forma y la configuración" (

49 Sin embargo, esa virtud, que no va ser otra cosa que vivir cristianamente, no puede ser satisfecha por cualquier hombre, sino por el sacerdote: así, el poder político se coloca bajo el poder papal.

50 Jürgen Habermas, Teoría y praxis, ob. cit., p. 55.

51 Ibid., p. 56.

52 La resemantización del concepto ha sido destacada principalmente por Francisco BERTELLONI, "Facere de necessitate virtutem", ob. cit., pp. 9-39.

$531014 \mathrm{~b}$.

54 1015a. 
una vez que cumple su télos. Así pues, dice que la phýsis es "la entidad, es decir, la forma” (

También en la Fisica, Aristóteles presenta esta relación de la naturaleza con la forma o la esencia propia de cada cosa. Define la phýsis como la materia que subyace en cada cosa que tenga en sí misma el principio del movimiento y del cambio, y también dice que "es la forma o la especie según la definición" (†

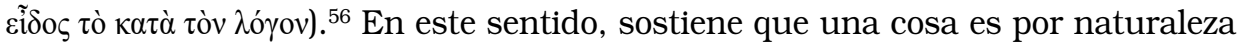
no cuando está en potencia (dýnamis), sino cuando es en acto (enérgeia) y alcanza así la forma específica de su definición: "cada cosa es lo que es cuando existe

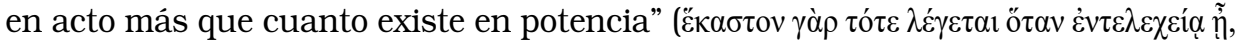

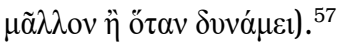

Este breve desarrollo nos permite ver cómo el enfoque teleológico de Aristóteles supone que la realización plena de la naturaleza de la pólis se logra cuando alcanza la plenitud a la que tiende, esto es, su télos (el vivir bien); y este fin se explicita al realizar plenamente su forma o esencia, a la cual tiende por naturaleza. Si tomamos en cuenta la Ética Nicomáquea, hay que agregar que ese fin supone el bien y lo mejor. Esto mismo lo sostiene en la Física, cuando dice que "aquello para lo cual las cosas son, tiende a ser lo mejor y su fin” (

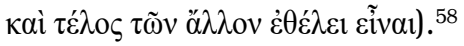

La concepción política de la naturaleza, entendida como realización de perfecciones ético-racionales, es lo que se abandona en el medioevo. La traducción latina de la Politica realizada por Guillermo de Moerbeke, aunque seguramente trató de ser lo más fiel al texto griego, no pudo evitar que la expresión "homo natura civile animal est" 59 sea interpretada con amplitud y con un sentido distinto al aristotélico. Esto obedece al hecho de que en el siglo XIII la natura tenía una semántica distinta a la phýsis griega, y es precisamente lo que se puede advertir en los escritos de Tomás de Aquino. El Aquinate habla de que "el hombre es por naturaleza un animal politico y social" (naturale autem est homini ut sit animal sociale et politicum) ${ }^{60}$ o que "es natural al hombre vivir en sociedad de muchos" (est homini naturale ut societate multorum vivat). En estos usos, la natura no es aquella phýsis teleológica y virtuosa de Aristóteles; es más bien una naturaleza deficiente y que opera como causa eficiente cuyo efecto es la societas. En este sentido, el regnum no es un espacio de perfección del hombre ni tampoco, como causa final, es ontológicamente anterior a las necesidades de la vida. Al contrario, en virtud de la resemantización que opera en el lenguaje político medieval, el regnum es un momento posterior que viene a suplir una situación de falencia y conflicto en la

\begin{tabular}{ll}
\hline 55 & 1015a. \\
56 & $193 \mathrm{a}$. \\
57 & $193 \mathrm{~b}$. \\
58 & $195 \mathrm{a}$. \\
59 & Texto de la edición de Franz Suseminl, Aristotelis Politicorum libri octo: cum vetusta translatione \\
& Guilelmi de Moerbeka, Leipzig, B. G. Teubneri, 1872, p. 7. \\
& De regno, lib. I, cap. 1.
\end{tabular}


societas. ${ }^{61}$ Así pues, el fundamento último del origen del regnum es la naturaleza falente y conflictiva del hombre: la natura tomista es una devaluación del naturalismo político de Aristóteles.

Por lo demás, no se puede pensar que este cambio se haya producido como consecuencia de una mera yuxtaposición de ideas clásicas y medievales. Esta mirada, además de pobre, no lograría explicar cómo los nuevos horizontes conceptuales alteran los viejos desde su interior. En realidad, la resemantización de las categorías clásicas que se advierte en los textos de Tomás de Aquino se explica porque también cambiaron las condiciones de posibilidad para que él haya podido decir lo que dijo. ${ }^{62} \mathrm{El}$ nuevo contexto de enunciación está ligado al surgimiento de un profundo interés de justificar el orden político más allá de Dios. En efecto, recordemos que el gran conflicto de la teoría política medieval es la cuestión de dos soberanias, cada una con fines propios: el poder temporal y el poder espiritual. ${ }^{63}$ La cuestión se remonta a los comienzos del siglo IV, cuando Constantino se convierte al cristianismo y funda Constantinopla en Bizancio. ${ }^{64} \mathrm{El}$ hito fue de suma importancia pues, por un lado, otorgaba al Imperio una nueva capital, limpia de la sangre cristiana que se había derramado en la vieja Roma pagana y, por el otro, permitió que se estableciera la doctrina de que el emperador cristiano era el representante directo de Dios en la tierra. Así pues, en el Imperio bizantino primaba la concepción unitaria del poder según la cual no había distinción entre el poder espiritual y el temporal. Sin embargo, tras la caída del Imperio romano de Occidente en el 476, la Iglesia del obispo de Roma se fortaleció, pudo transformarse en la heredera política del viejo imperio y estableció la idea de que el poder espiritual tenía primacía sobre el temporal. ${ }^{65}$ Se erigió, pues, una monarquía papal como una contrafigura del unitarismo bizantino. De hecho, esa plena potestas es ratificada por la donatio Constantini. ${ }^{66}$ Recordemos que, según esta falsificación, ${ }^{67}$ el papa es puesto en las funciones del emperador mediante la cesión de todos los derechos y poderes temporales realizada por el propio Constantino.

61 Francisco Bertelloni, "Natura multipliciter dicitur”, ob. cit., p. 22.

62 En este sentido, Bertelloni señala tres hechos fundamentales que posibilitaron el surgimiento de un nuevo pensamiento político medieval: en primer lugar, una nueva orientación que se produjo con la introducción de la Ética Nicomáquea y la Política de Aristóteles; en segundo lugar, el paso de la teologia política a la filosofía política, la cual se verifica con la aparición del nuevo maestro urbano que irrumpió en el escenario intelectual; finalmente, la disposición a renovar las ideas por parte de los autores medievales (Francisco BERTELLONI, "El surgimiento de la scientia política en el siglo XIII", en Hugo ZuRUTUZA et al. (comps.), El hilo de Ariadna. Del tardoantiguo al tardomedioevo, Rosario, Homo Sapiens, 1996, pp. 178-179.

63 Ya en el siglo XIII, con la irrupción de la Ética Nicomáquea, la doctrina Aristotélica de que los actos humanos se realizan en vistas a un fin y un fin último sirvió para justificar un fin análogo a cada uno de esos poderes.

64 En esta breve descripción histórica seguimos en gran parte el trabajo de Francisco BertelLONI, “¿El destino del Estado, coincide o no con el de sus dioses?, en Anales de historia antigua, medieval y moderna, 37-38 (2005), pp. 105-118.

65 En relación con el surgimiento del papado romano como reacción contra Bizancio, ver Ernst BENZ, The Eastern Orthodox Church: Its Thought and Life, New York, Routledge, 2017, pp. 175-183

66 Sobre la donación de Constantino ver el trabajo de Johannes Fried, Donation of Constantine and Constitutum Constantini: The Misinterpretation of a Fiction and Its Original Meaning, Berlin, Walter de Gruyter, 2007.

67 Lorenzo Valla fue quien demostró con argumentos sólidos que la donación era un engaño, mediante su trabajo De falso credita et ementita Constantini Donatione declamatio, escrito entre 1439 y 1440. 
La primacía del poder espiritual es precisamente lo que Aristóteles y los estoicos ayudaron a desarmar en el siglo XIII. La gran novedad medieval es, pues, el proceso de secularización del Estado: se produce a partir del uso de categorias aristotélicas que permiten entender el poder temporal y fundamentarlo. En el caso de la fundamentación del poder espiritual está claro que Cristo había venido con una misión redentora y había otorgado a ciertos encargados que llevasen a cabo esa tarea: el papa es el gran administrador de los fines espirituales del hombre que constituyen la salvación. En el caso del poder temporal es donde se empieza a diseñar un modo propio de justificación con las nuevas categorías filosóficas que irrumpieron en la época. La labor de Tomás de Aquino, que hemos estudiado, se enmarca y se comprende dentro de este desplazamiento del lenguaje político y la necesidad de teorizar acerca del surgimiento del orden politico del poder omniabarcador de la Iglesia.

\section{CONCLUSIONES}

Ullmann explica que en el periodo medieval anterior al siglo XIII el concepto de Estado como conjunto independiente, autosuficiente y autónomo de ciudadano era por completo desconocido. Recién en el siglo XIII hizo su aparición como consecuencia del pensamiento de Aristóteles. ${ }^{68}$ La influencia que produjo el filósofo griego causó una verdadera transmutación en el pensamiento de la época, que el propio Ullmann califica como "revolución conceptual". ${ }^{69}$ En este orden de ideas, en el marco más amplio de la teoría de los dos poderes distintos (el poder temporal y el poder espiritual), la irrupción de la Ética Nicomáquea y la Política de Aristóteles en el Medioevo, sumado al pensamiento estoico ya conocido en el ámbito universitario, permitió que Tomás de Aquino ideara un tipo de justificación propia del orden estatal al margen de la Iglesia, mediante la cual le atribuyó dignidad ontológica. Sin embargo, en esta empresa operaba una inconsciente resemantización de las categorías políticas clásicas que posibilitó una fundamentación del origen del Estado con base en un modelo de necesidades y falencias. En esta concepción, el Estado encuentra su origen en la autoconservación individual y se constituye en un ámbito donde lo privado prima por sobre lo público, y donde los vínculos de dominio son necesarios para remediar los conflictos entre particulares.

Hemos visto que Arendt y Habermas se han ocupado de indagar este desplazamiento y han demostrado cómo Tomás de Aquino abandona el modelo virtuoso aristotélico y confunde el oîkos con la pólis y los vínculos de dominio en cada esfera. Sin embargo, hemos podido ver, luego de nuestro estudio, que en ese nuevo diseño del Estado protomoderno existe un concepto fundamental que también ha variado: la naturaleza. El naturalismo aristotélico supone un compromiso esencial de la phýsis con el télos, la forma y el bien. El alcance de la teleología en Aristóteles es más abarcador que en el caso de los medievales y mucho más virtuoso. Al ser

68 Walter Ullmann, Historia del pensamiento político en la Edad Media, Barcelona, Ariel, 2013, p. 19.

69 Ibid., p. 152. 
el hombre político por naturaleza está comprometido con el finalismo de la pólis y la realización de la areté.

En el caso de Tomás de Aquino hay un viraje hacia el principio de que el hombre tiende a la autoconservación. Así pues, lo finalístico no es el Estado sino la autoconservación, que es la razón por la que se instituye el orden político. La prioridad que el filósofo griego le daba al télos y el buen vivir es sustituida en Tomás de Aquino por un discurso que privilegia el origen, es decir, las necesidades naturales como fundamento de la constitución del regnum. Entre la phýsis aristotélica y la natura tomista solo queda en común el nombre.

La resemantización de la phýsis a la natura permitió la sustitución del modelo político virtuoso y el diseño de un modelo del Estado protomoderno que acentúa las necesidades biológicas. La importancia que implica este análisis para la filosofia y la historia de la teoría política es que precisamente demuestra cómo la naturaleza que invoca Tomás de Aquino anticipa aquella condición que sostendrán los filósofos modernos y prepara el terreno para el desarrollo de sus teorías acerca del origen del Estado.

\section{BIBLIOGRAFÍA}

\section{Ediciones y traducciones de textos griegos y latinos}

Barbado Viejo, Francisco (dir.), Suma Teológica de Santo Tomas de Aquino, edición bilingüe, 16 vols., Madrid, La Editorial Católica, 1956.

Burnet, John (ed.), Respublica. Platonis Opera, tomos IV-V, Oxford, Clarendon Press, 1905-1907.

Bywater, Ingram (ed.), Aristotelis. Ethica Nicomachea, Oxford, Clarendon Press, 1988.

JAEger, Werner (ed.), Aristotelis Metaphysica, Oxford, Clarendon Press, 1957.

Marías, Julián y María Araúso (eds. y trads.), Aristóteles. Política, Madrid, Institutos de Estudios Políticos, 1951.

RackHAm, Harris (ed. y trad.) Cicero. De Finibus Bonorum et Malorum, London, Heinemann, 1931.

Ross, William David (ed.), Aristole’s Physics, Oxford, Clarendon Press, 1936.

Suseminl, Franz (ed.), Aristotelis Politicorum libri octo: cum vetusta translatione Guilelmi de Moerbeka, Leipzig, B. G. Teubneri, 1872.

Tursi, Antonio (trad.), Tomás de Aquino, Del reino, Buenos Aires, Losada, 2003.

\section{Bibliografia secundaria}

Arendt, Hannah, La condición humana, Buenos Aires, Paidós, 2009 (primera edición 1958). 
ARGÜELLo, Santiago, "El lenguaje de la causalidad de nuestras acciones: hacia una resignificación del dominus en Tomás de Aquino”, Pensamiento 71 (267) (2015), pp. 565-586. DOI: https://doi.org/10.14422/pen.v71.i267.y2015.002

BaIlly, Anatole, Dictionnaire Grec-Français, Paris, Hachette, 2000.

Benz, Ernst, The Eastern Orthodox Church: Its Thought and Life, New York, Routledge, 2017.

Bertelloni, Francisco, “¿El destino del Estado, coincide o no con el de sus dioses?, en Anales de Historia Antigua, Medieval y Moderna 37-38 (2005), pp. 105-118. Recuperado de http://revistascientificas.filo.uba.ar/index.php/analesHAMM/ article/view/3513

Bertelloni, Francisco, “¿Qué significa 'politica’ en STh. I, q. 96, a. 4? Sobre la génesis de la semántica de un vocablo”, en José Meirinhos y Olga WeIJERs (eds.) Florilegium mediaevale. Études offertes à Jacqueline Hamesse à l'occasion de son éméritat (col. Textes et Études du Moyen Âge, 50), Louvain-La-Neuve, 2009, pp. 19-37.

Bertelloni, Francisco, "Algunas reinterpretaciones de la causalidad final aristotélica en la teoría política medieval", Cadernos de Historia e Filosofia da Ciencia, 15 (2) (2005), pp. 343-372. Recuperado de https://www.cle.unicamp.br/ eprints/index.php/cadernos/article/view/620

Bertelloni, Francisco, "El surgimiento de la scientia politica en el siglo XIII", en Hugo Zurutuza, et al. (comps.), El hilo de Ariadna. Del tardoantiguo al tardomedioevo, Rosario, Homo Sapiens, 1996, pp. 178-179.

Bertelloni, Francisco, "Facere de necessitate virtutem. El principio conservatio sui en la teoría politica medieval”, en Devs mortales, 11 (2015), pp. 9-39.

Bertelloni, Francisco, "Natura multipliciter dicitur. Variantes en el uso de natura en la teoría politica medieval a partir de la segunda mitad del siglo XIII", en Scripta 4 (2) (2011), pp. 11-29. Recuperado de http://revistas.uncu.edu.ar/ ojs/index.php/scripta/article/view/395

Chappell, Timothy, “'Naturalism' in Aristotle's Political Philosophy," en Ryan BALOT (ed.), A Companion to Greek and Roman Political Thought, Oxford, WileyBlackwell, 2009, pp. 382-398.

Corso DE EstradA, Laura, "Oikeíosis. Ciceronian Reading and XIII Century Receptions”, en Alejandro Vigo (ed.), Oikeiosis and the Natural Basis of Morality. From Classical Stoicism to Modern Philosophy, Hildesheim, G. Olms, 2012, pp. 67-94.

Engberg-Pedersen, Troels, The Stoic Theory of Oikeiosis: Moral Development and Social Interaction in Early Stoic Philosophy, Aarhus, University Press, 1991.

Fortin, Ernest, “St. Thomas Aquinas”, en Leo Strauss y Joseph Cropsey (eds.), History of Political Philosophy, Chicago, University Press, 1987, pp. 248-275.

FRIED, Johannes, Donation of Constantine and Constitutum Constantini: The Misinterpretation of a Fiction and Its Original Meaning, Berlin, Walter de Gruyter, 2007. 
Giuseppina, Magnaldi, L'oikcíwors peripatetica in Ario Didimo e nel De finibus di Cicerone, Florencia, Le Lettere, 1991.

Habermas, Jürgen, Teoría y praxis, Madrid, Tecnos, 1987.

HobBes, Thomas, Leviatán: o la materia, forma y poder de una república eclesiástica y civil, Buenos Aires, FCE, 2007 (primera edición 1651).

Kullmann, Wolfgang, "Man as a political animal in Aristotle”, en David Keyt y Fred Miller, Jr. (eds.), A Companion to Aristotle's Politics, Oxford, Blackwell, 1991, pp. 94-117.

LidDELl, Henry George y Robert ScotT, A Greek-English Lexicon, Oxford, Clarendon Press, 1996.

Locke, John, Second Treatise of Government, Indianapolis, C.B. Macpherson, 1980 (primera edición 1690).

Magnavacca, Silvia, Léxico técnico de filosofia medieval, Buenos Aires, Miño y Dávila, 2014.

MÁrsico, Claudia, "El status de la pólis sana en La República de Platón”, en Diálogos 74 (1999), pp. 149-167. Recuperado de https://www.academia. edu/5276428/El_status_de_la_p\%C3\%B3lis_sana_en_la_Rep\%C3\%BAblica_ de_Plat\%C3\%B3n

MaYhew, Robert, Aristotle's Criticism of Plato's Republic, Lanham, Rowman \& Littlefield, 1997.

ReEve, Christopher, "The naturalness of the polis in Aristotle," en Georgios AnAGnostopoulos (ed.), A Companion to Aristotle, Oxford, Wiley-Blackwell, 2009, pp. 512-525.

SOAREs, Lucas, Platón y la política, Madrid, Tecnos, 2010.

Ullmann, Walter, Historia del pensamiento político en la Edad Media, Barcelona, Ariel, 2013. 